Published in final edited form as:

Gynecol Oncol. 2009 August ; 114(2): 195-198. doi:10.1016/j.ygyno.2009.04.012.

\title{
IMPACT ON SURVIVAL OF 12 VERSUS 3 MONTHLY CYCLES OF PACLITAXEL (175 MG/M²) ADMINISTERED TO PATIENTS WITH ADVANCED OVARIAN CANCER WHO ATTAINED A COMPLETE RESPONSE TO PRIMARY PLATINUM-PACLITAXEL: FOLLOW-UP OF A SOUTHWEST ONCOLOGY GROUP AND GYNECOLOGIC ONCOLOGY GROUP PHASE 3 TRIAL
}

\author{
Maurie Markman ${ }^{1}$, PY Liu ${ }^{2}$, James Moon ${ }^{2}$, Bradley J. Monk ${ }^{3}$, Larry Copeland ${ }^{4}$, Sharon \\ Wilczynski $^{5}$, and David Alberts ${ }^{6}$ \\ ${ }^{1}$ University of Texas MD Anderson Cancer Center, Houston, TX \\ 2 Southwest Oncology Group Statistical Center, Seattle WA \\ 3 University of California Irvine Medical Center, Orange, CA \\ ${ }^{4}$ Ohio State University Health Center, Columbus, $\mathrm{OH}$ \\ ${ }^{5}$ City of Hope National Medical Center, Duarte, CA \\ ${ }^{6}$ University of Arizona Cancer Center, Tucson, AZ
}

Abstract

Objectives-A SWOG/GOG phase 3 trial exploring the impact of 12-monthly cycles of paclitaxel given to patients with advanced ovarian cancer who achieved a complete response to primary chemotherapy was discontinued by the Data Safety and Monitoring Committee when a prospectivelydefined interim analysis revealed a highly statistically significant improvement in progression-free survival (PFS). At study closure, it was too early to assess the impact on overall survival.

Methods-Patients $(\mathrm{n}=296)$ received either 3 or 12 monthly cycles of paclitaxel $\left(175 \mathrm{mg} / \mathrm{m}^{2}\right.$ over 3-hours).

Results-Of the 146 patients on the 3-cycle arm, 9 (6\%) received > 3-cycles. Median (12 versus 3 cycle; intention-to-treat analysis) updated PFS (all pts) 22 versus 14 months, $\mathrm{p}=0.006$; overall survival (all pts) 53 versus 48 months, $\mathrm{p}=0.34$.

Conclusion-Twelve cycles of single agent maintenance paclitaxel significantly improves PFS. Explanations for the lack of a favorable influence on overall survival include: (a) treatment at relapse equalized outcome; (b) the sample size was insufficient to reveal a difference; (c) "crossover" of

Address for correspondence: Maurie Markman, M.D, University of Texas MD Anderson Cancer Center, 1515 Holcombe Boulevard, Houston, TX, 77005, Phone: 713-745-7140; FAX: 713-563-9586; e-mail: mmarkman@ mdanderson.org.

Conflict of Interest Statement

The authors have no conflicts of interest to declare.

Publisher's Disclaimer: This is a PDF file of an unedited manuscript that has been accepted for publication. As a service to our customers we are providing this early version of the manuscript. The manuscript will undergo copyediting, typesetting, and review of the resulting proof before it is published in its final citable form. Please note that during the production process errors may be discovered which could affect the content, and all legal disclaimers that apply to the journal pertain. 
patients from 3 cycles to longer treatment masked a potential difference. An ongoing phase 3 trial will hopefully provide a definitive answer to the question of the impact of this maintenance strategy on overall survival.

\section{Introduction}

In an effort to improve the survival of women with advanced ovarian cancer who have been shown to be without clinical evidence of disease following the completion of primary platinumpaclitaxel chemotherapy, the Southwest Oncology Group (SWOG) and the Gynecologic Oncology Group (GOG) initiated a randomized phase 3 trial which explored the potential efficacy of administering an additional year (12 monthly cycles) of single agent paclitaxel in this setting [1].

The rationale for this investigative strategy included: (a) potential for clinically-relevant continued tumor cell kill for this known cycle-specific cytotoxic agent [2-4]; (b) theorized anti-angiogenic properties of paclitaxel [5]; (c) demonstrated safety associated with delivery of the agent for $>1$ year, with no reports of secondary malignancies or severe organ dysfunction (with the exception of peripheral neuropathy) developing due to "chronic use" [6-8]; and (d) limited retrospective (non-randomized) data suggesting prolonged paclitaxel administration in the second-line setting may be capable of substantially delaying subsequent disease progression for extended periods [6].

When a prospectively defined interim analysis of this study (SWOG-9701/GOG-178) performed upon entry of approximately one-half of the planned patient population revealed "extreme differences" in progression-free survival (PFS) in favor of the administration of 12 monthly cycles of single agent paclitaxel, compared to 3-cycles (median PFS 28 months versus 21 months, $\mathrm{p}=0.0023$; HR 0.43), the SWOG Data Safety and Monitoring Committee (DSMC) elected to close the trial and inform all patients of the outcome [1]. At the time of closure of this trial there were an insufficient number of deaths $(n=17)$ to draw any conclusions regarding the potential impact of extended paclitaxel therapy on overall survival in this clinical setting.

With the passage of several years since the closure of this provocative trial, and with the occurrence of additional deaths, it is appropriate to now report the observed impact on overall survival associated with this novel management strategy.

\section{Materials and Methods}

\section{Study design}

The patient population, eligibility criteria, treatment schema, and dosage modifications for this trial, as well as the toxicity profile associated with extended paclitaxel therapy (12-monthly treatments) have previously been described in detail [1]. Therefore, in this manuscript, the study design will only be outlined in brief.

Patients eligible for entry into this multi-center randomized phase 3 trial had to have a histologically-confirmed diagnosis of advanced ovarian cancer or primary carcinoma of the peritoneum. They were required to have attained a clinically-defined complete response to 56 cycles of a primary platinum (either cisplatin or carboplatin) plus paclitaxel chemotherapy regimen (e.g., normal CT scan of the abdomen/pelvis, normal physical examination, CA-125 antigen level $\leq 35$, no symptoms suggestive of the presence of active disease). Individuals with a neuropathy of > grade 1 in severity following the primary chemotherapy regimen were not permitted entry into this trial. 
Patients were randomized to receive either 3 or 12-cycles of single agent paclitaxel, delivered at a dose of $175 \mathrm{mg} / \mathrm{m}^{2}$ (infused over 3-hours) on an every 28-day schedule. Patients experiencing excessive toxicity would have treatment delayed for a maximum of 2 weeks until the side effect had resolved to baseline, with subsequent paclitaxel cycles delivered at a dose of $135 \mathrm{mg} / \mathrm{m}^{2}$. Patients developing a grade 3 neuropathy had treatment discontinued.

(Note: Due to a somewhat higher "drop-out rate" on the 12-cycle study arm, not specifically due to the development of treatment-related side effects, the study was modified to lower the initial dose of paclitaxel in both arms to $135 \mathrm{mg} / \mathrm{m}^{2}$. This treatment amendment became operational only a few months prior to the trial's permanent closure by the DSMC. Thus, very few patients entered into this trial actually had therapy initiated at this lower dose level.)

\section{Statistical considerations}

The study was originally designed with progression-free survival as the primary endpoint and the sample size goal was 450 patients [1]. Should there be a true 12 versus 3 -cycle death hazard ratio of 0.67 for overall survival (i.e. a 50\% increase in median survival), 191 events would be needed to detect a statistically significant difference with a 0.80 power. There were 164 events at the time of the current analysis and the power to detect the above difference is approximately $75 \%$.

However, as a result of the early enrollment termination and a decreasing event rate, three or more additional years of follow-up would be needed to reach the 191-event count. Due to the understandable major interest in the oncology community in the potential implications of the highly favorable progression-free survival results of this trial, a decision was made to analyze and publish the data at this time in order to provide some information regarding the impact of this management strategy on overall survival.

It should also be noted that it is highly unlikely that the survival outcomes reported here will change with additional follow-up. Based on simulations $(n=1000)$, if the observed hazard rates continue over the next 3 years, then the conditional power to detect a $50 \%$ increase in median overall survival (with $\mathrm{p} \leq 0.05$ ) is $4 \%$ (95\% CI: $3-5 \%$ ). However, if over the next 3 years the observed death hazard ratio between the arms is 0.67 among the remaining patients, then the conditional power is $26 \%$ (95\% CI: $23 \%-29 \%$ ). Finally, assuming a death hazard ratio of 0.5 over the next 3 years, the conditional power is $56 \%$ (95\% CI: 53-59\%).

To maintain the randomization balance, all patients are analyzed according to the intent-totreat principle (i.e., by randomized treatment assignment). Both unadjusted analysis and Cox model analysis were performed. The latter took into account randomization stratification factors: prior paclitaxel schedule ( $<24$ hours versus $\geq 24$ hours), disease state at diagnosis (optimal stage III, suboptimal stage III, stage IV), and age ( $\leq 65$ versus $>65$ ).

All $\mathrm{p}$ values included in this report are 2-sided.

Based on recently reported highly provocative findings of 'normal range' CA-125's predictive ability for PFS [9], exploratory analyses adding baseline CA-125 to the Cox model were conducted.

\section{Results}

A total of 308 patients enrolled to this multi-center cooperative group trial when it closed on December 1, 2001 [1]. Twelve patients were found to be ineligible by central review. The remaining 296 patients (146 on the 3-cycle arm and 150 on the 12-cycle arm) were evaluable. Of these 296 evaluable patients, 294 had baseline CA-125 data available. 
Protocol deviation was recorded for one patient randomized to the 12-cycle arm who refused all protocol treatment. In addition, subsequent to the DSMC's decision for early study termination and disclosure of PFS results, nine patients $(6 \%)$ on the 3-cycle arm continued treatment beyond the 3 cycles. These ten patients were excluded from toxicity evaluations.

Serious toxic events observed on this protocol are outlined in Table 1. There was a higher incidence of grade 2 and 3 neuropathy, and grade 3 pain (presumably due to neuropathy) in the 12-cycle treatment arm.

In an updated analysis, patients treated with 12 cycles of single agent paclitaxel experienced a statistically significant improvement in PFS (median: 22 months versus 14 months; unadjusted $\mathrm{p}$ value $=0.006)$ (Figure 1$)$.

In a Cox model analysis adjusting for stratification factors, the 12 versus 3-cycle treatment hazard ratio for PFS is $0.68(\mathrm{p}=0.004)$. The same hazard ratio is $0.70(\mathrm{p}=0.01)$ when baseline CA-125 is added to the model. In an exploratory analysis there was no indication that the magnitude of this treatment effect was different between the baseline CA-125 groups (interaction $\mathrm{p}=0.59$ ).

The median overall survival is 53 months for the12-cycle arm and 48 months for the 3-cycle arm with an unadjusted $\mathrm{p}$ value of 0.34 (Figure 2). In a Cox model analysis adjusting for stratification factors, the 12 versus 3 -cycle treatment hazard ratio is $0.88(\mathrm{p}=0.40)$. The same hazard ratio is $0.96(\mathrm{p}=0.78)$ when baseline CA-125 is added to the model.

However, in an exploratory subset analysis by Cox models adjusting for stratification factors, the 12-cycle versus 3-cycle hazard ratio is $0.65(\mathrm{p}=0.07)$ for patients with baseline CA-125 < $10(\mathrm{n}=171)$. In this subset, the median survival was 79 months for patients on the 12-cycle arm versus 54 months for patients on the 3-cycle arm. In the subset of patients with CA-125>10 $(\mathrm{n}=123)$, the 12-cycle versus 3 -cycle hazard ratio is $1.27(\mathrm{n}=0.28)$ with median overall survival estimates of 40 months and 46 months, respectively. Furthermore, the interaction term between treatment and CA-125 had a p-value of 0.06. Although it must be stressed that this was merely an exploratory analysis, it suggests a possible differential treatment effect in terms of overall survival between the baseline CA-125 groups.

\section{Discussion}

Multiple attempts to improve the survival of patients with solid tumors by extending the duration of treatment, including with platinum-based chemotherapy in advanced ovarian cancer, have generally (but not universally) failed to demonstrate clinical utility of this management approach [10-13].

Thus, the initial finding of a significant impact of 12 cycles of single agent monthly paclitaxel on the median PFS (7 months) in advanced ovarian cancer in women who had attained a clinically-defined complete response to a regimen which included paclitaxel was met, by some, with genuine disbelief [1]. For example, it was stated by several commentators that the initial analysis of PFS was based on a total of only 54 events. Overall, there are approximately four times as many PFS events now (224) as there were in the initial analysis (54). PFS between the study arms continues to be highly statistically significant.

Others expressed a feeling that even with this substantial modification in the pattern of disease recurrence, such treatment was not justified in the absence of a documented improvement in overall survival, due to the potential toxicities (principally neuropathy) associated with this therapeutic regimen. That subsequently reported ovarian cancer studies, with somewhat different designs compared to the current trial, failed to reveal any benefit associated with 
administering single agent topotecan after the completion of a primary platinum/paclitaxel chemotherapy program has only heightened concern for the apparent uniqueness of the effects associated with "maintenance" paclitaxel [14].

It should also be noted that another ovarian cancer randomized single agent paclitaxel maintenance chemotherapy trial, with a smaller patient population (approximately 100 women in a "clinically-defined complete response") and a shorter duration of therapy (every 3-weeks for 6 cycles) failed to reveal evidence of improvement in outcome associated this strategy [15].

What can be concluded from this analysis of survival from the SWOG-9701/GOG-178 study? As predicted by many, it is unfortunately clear that the total sample size available for an evaluation of overall survival is simply inadequate to make any definite statements regarding the impact of this management approach. This analysis is confounded by the fact a number of patients $(6 \%)$ randomized to the 3 -cycle arm actually received more prolonged (> 3 cycles) treatment with paclitaxel, based on the reported results leading to closure of the study by the DSMC.

It is relevant to note here that the GOG is conducting a confirmatory trial for the current study. The importance of this effort in further documenting both the impact of taxane "maintenance" therapy on PFS, and (most importantly) overall survival in advanced ovarian cancer, cannot be overstated.

Of interest, an exploratory analyses performed on the data generated from this trial, but not prospectively defined when this study was designed, regarding the impact of extended paclitaxel treatment for patients with a baseline (pre-maintenance therapy) CA-125 antigen level of $\leq 10$ is potentially of clinical relevance. As Figure 3 shows, even within the traditionally regarded 'normal' range (i.e. $\leq 35$ ), baseline CA-125 is highly predictive of survival (Cox model $\mathrm{p}=0.01$ including treatment and stratification factors).

It is reasonable to speculate the patient population with baseline CA-125 antigen levels $\leq 10$ had the lowest total body burden of residual malignant cells. These data support the provocative concept that 12 additional monthly cycles of single agent paclitaxel may be sufficient to produce a major favorable impact on survival (both progression-free and overall) with this extent of persistent microscopic and macroscopic cancer, but not when larger volumes are present. It will be important to evaluate this specific issue in the ongoing GOG paclitaxel maintenance chemotherapy trial. Of course, it is relevant to also acknowledge that patients with the lowest baseline CA-125 levels experience a superior outcome, independent of the subsequent delivery of any maintenance strategy (9).

Finally, how might the survival results (both PFS and overall) from this phase 3 trial be utilized by oncologists, and their patients with advanced ovarian cancer who are found to have achieved a clinically-defined complete response to platinum-taxane chemotherapy? If a patient has not experienced excessive toxicity from primary treatment (e.g., grade 2-3 neurotoxicity), and she wishes to consider a management option to prolong the time to disease progression (both objective and symptomatic), and possibly improve overall survival, it is appropriate to discuss with her the following:

a. This multi-center randomized study has shown that the administration of up to 12cycles of single agent paclitaxel delivered on a monthly schedule may significantly delay the time to disease progression.

b. The "cost" of continuing treatment will include alopecia until treatment is stopped, the risk of the initial development, or worsening, of a peripheral neuropathy, and the need for monthly office visits to receive intravenous paclitaxel. 
c. Unfortunately, based on existing data it is not possible to determine whether, or not, this management strategy will favorably impact overall survival.

\section{References}

1. Markman M, Liu PY, Wilczynski S, Monk B, Copeland LJ, Alvarez RD, et al. Phase III randomized trial of 12 versus 3 months of maintenance paclitaxel in patients with advanced ovarian cancer after complete response to platinum and paclitaxel-based chemotherapy: A Southwest Oncology Group and Gynecologic Oncology Group trial. J Clin Oncol 2003;21:2460-5. [PubMed: 12829663]

2. Rowinsky EK, Donehower RC. Paclitaxel (Taxol). N Engl J Med 1995;332:1004-14. [PubMed: 7885406]

3. Rowinsky EK, Donehower, Jones RJ, Tucker RW. Microtubule changes and cytotoxicity in leukemia cell lines treated with taxol. Cancer Res 1988;48:4093-100. [PubMed: 2898289]

4. Lopes NM, Adams EG, Pitts TW, Bhuyan BK. Cell kill kinetics and cell cycle effects of taxol on human and hamster ovarian cell lines. Cancer Chemother Pharmacol 1993;32:235-42. [PubMed: 8098996]

5. Browder T, Butterfield CE, Kraling BM, Shi B, Marshall B, O’Reilly MS, et al. Antiangiogenic scheduling of chemotherapy improves efficacy against experimental drug-resistant cancer. Cancer Res 2000;60:1878-86. [PubMed: 10766175]

6. Markman M, Hakes T, Barakat R, Curtin J, Almadrones L, Hoskins W. Follow-up of Memorial SloanKettering Cancer Center patients treated on National Cancer Institute Treatment Referral Center Protocol 9103:Paclitaxel in refractory ovarian cancer. J Clin Oncol 1996;14:796-9. [PubMed: 8622026]

7. von Gruenigen VE, Karlen JR, Waggoner SE. A case of chronic paclitaxel administration in ovarian cancer. Gynecol Oncol 2003;89:532-5. [PubMed: 12798725]

8. Rohl J, Kushner D, Markman M. Chronic administration of single-agent paclitaxel in gynecologic malignancies. Gynecol Oncol 2001;81:201-5. [PubMed: 11330950]

9. Markman M, Liu PY, Rothenberg ML, Monk BJ, Brady M, Alberts DS. Pretreatment CA-125 and risk of relapse in advanced ovarian cancer. J Clin Oncol 2006;24:1454-8. [PubMed: 16549840]

10. Hakes TB, Chalas E, Hoskins WJ, Jones WB, Markman M, Rubin SC. Randomized prospective trial of 5 versus 10 cycles of cyclophosphamide, doxorubicin, and cisplatin in advanced ovarian carcinoma. Gynecol Oncol 1992;45:284-9. [PubMed: 1612505]

11. Bertelsen K, Jakobsen A, Stroyer J, Nielsen K, Sandberg E, Andersen JE, et al. A prospective randomized comparison of 6 and 12 courses of cyclophosphamide, Adriamycin, and cisplatin in advanced epithelial ovarian cancer: A Danish Ovarian Study Group trial (DACOVA). Gynecol Oncol 1993;49:30-6. [PubMed: 8482557]

12. Lambert HE, Rustin GJS, Gregory WM, Nelstrop AE. A randomized trial of five versus eight courses of cisplatin or carboplatin in advanced epithelial ovarian carcinoma. Ann Oncol 1997;8:327-33. [PubMed: 9209661]

13. Bozcuk H, Artac M, Ozdogan M, Savas B. Does maintenance/consolidation chemotherapy have a role in the management of small cell lung cancer? Cancer 2005;104:2650-7. [PubMed: 16284984]

14. DePlacido S, Scambia G, DiVagno G, Naglieri E, Lombardi AV, Biamonte R, et al. Topotecan compared with no therapy after response to surgery and carboplatin/paclitael in patients with ovarian cancer: Multicenter Italian Trials in Ovarian Cancer (MITO-1) randomized study. J Clin Oncol 2004;22:2635-42. [PubMed: 15226331]

15. Conte PF, Favalli G, Gadducci A, Katsaros D, Benedetti Panici PL, Carpi A, et al. Final results of After-6 protocol 1: A phase III trial of observation versus 6 courses of paclitaxel in advanced ovarian cancer patients in complete response after platinum-paclitaxel chemotherapy. J Clin Oncol 2007;25 (18 suppl):275s.(abstract 5505) 


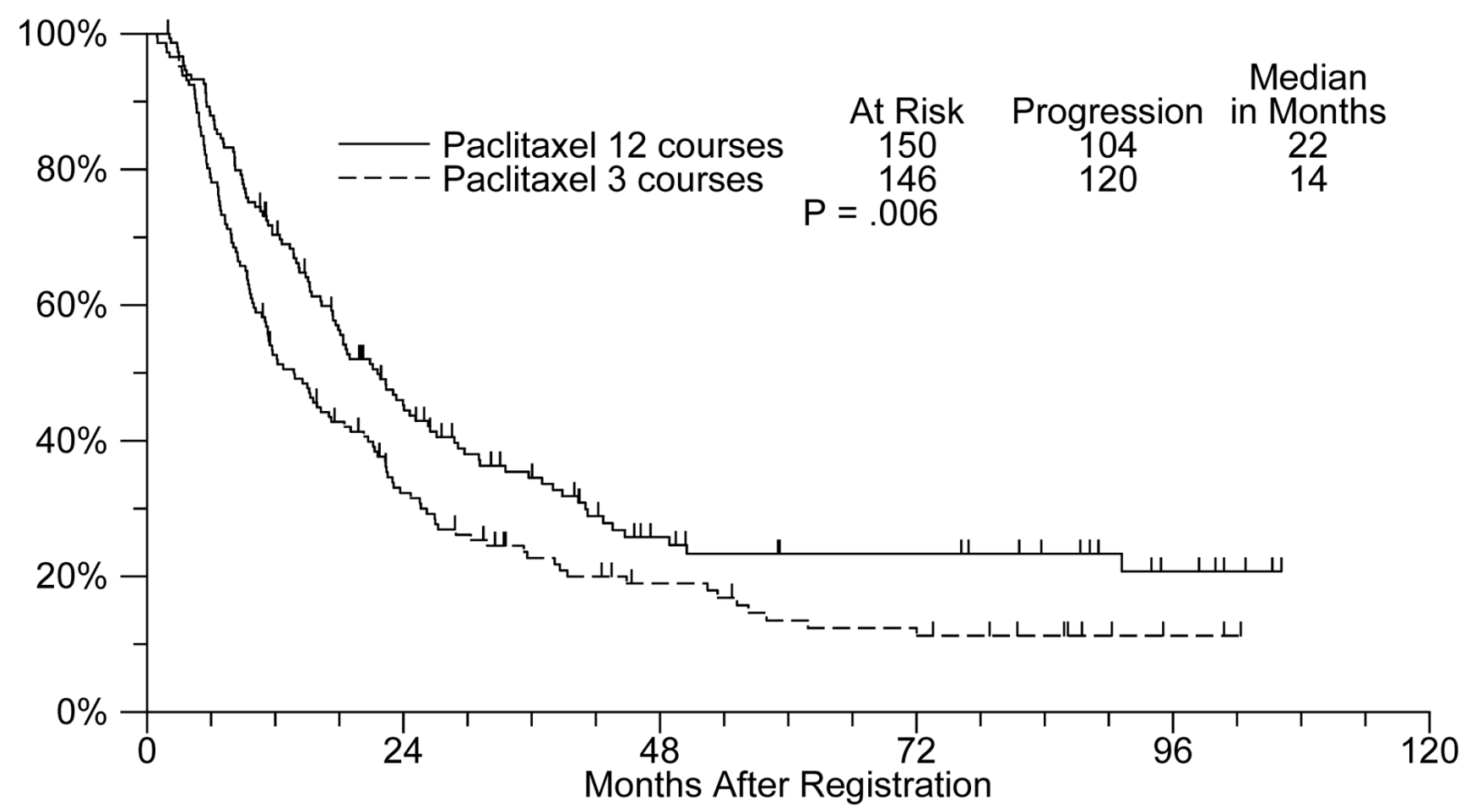

Figure 1.

Updated PFS (12 versus 3 cycles of single agent paclitaxel) 


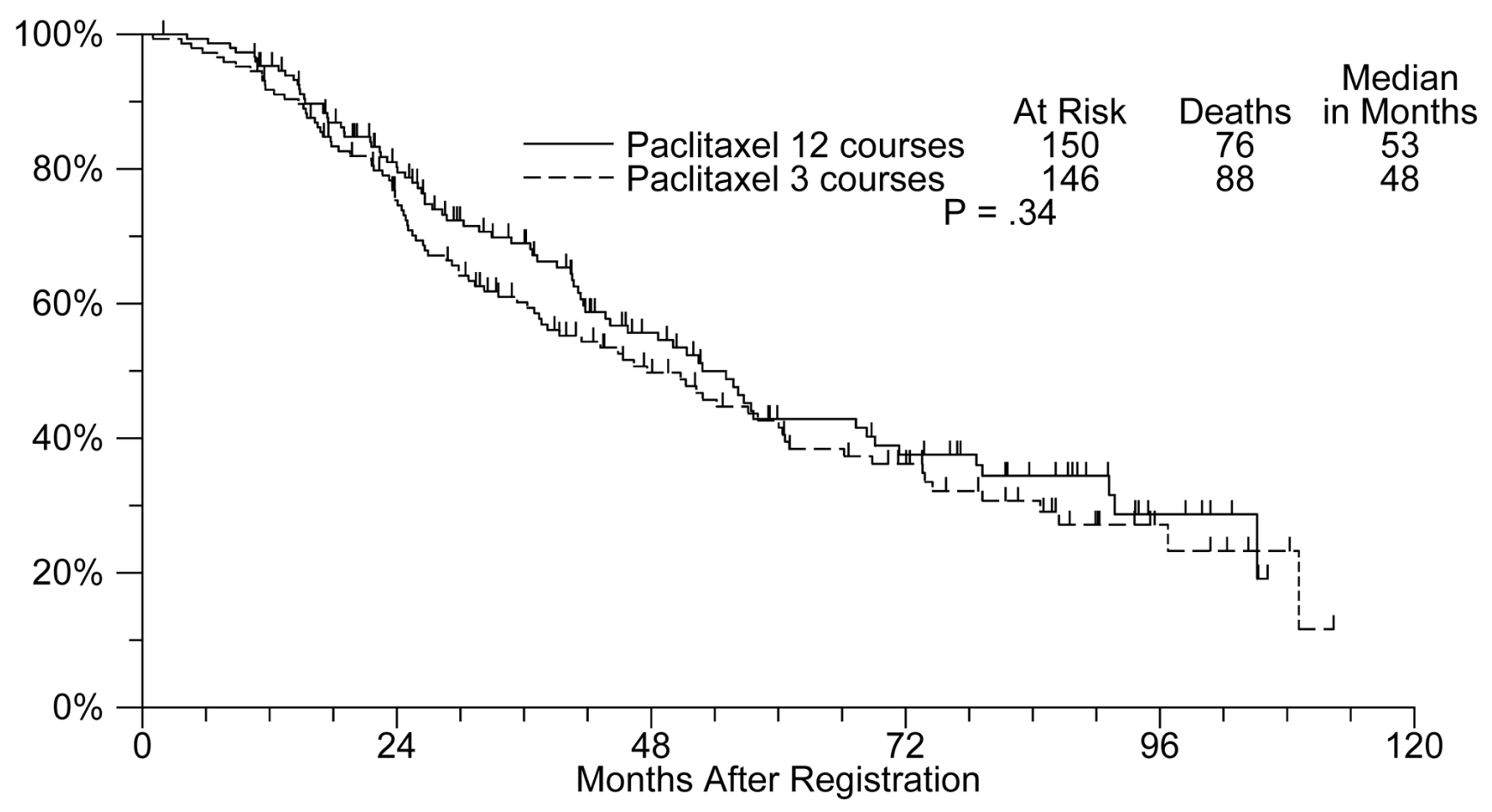

Figure 2.

Overall survival (12 versus 3 cycles of single agent paclitaxel) 


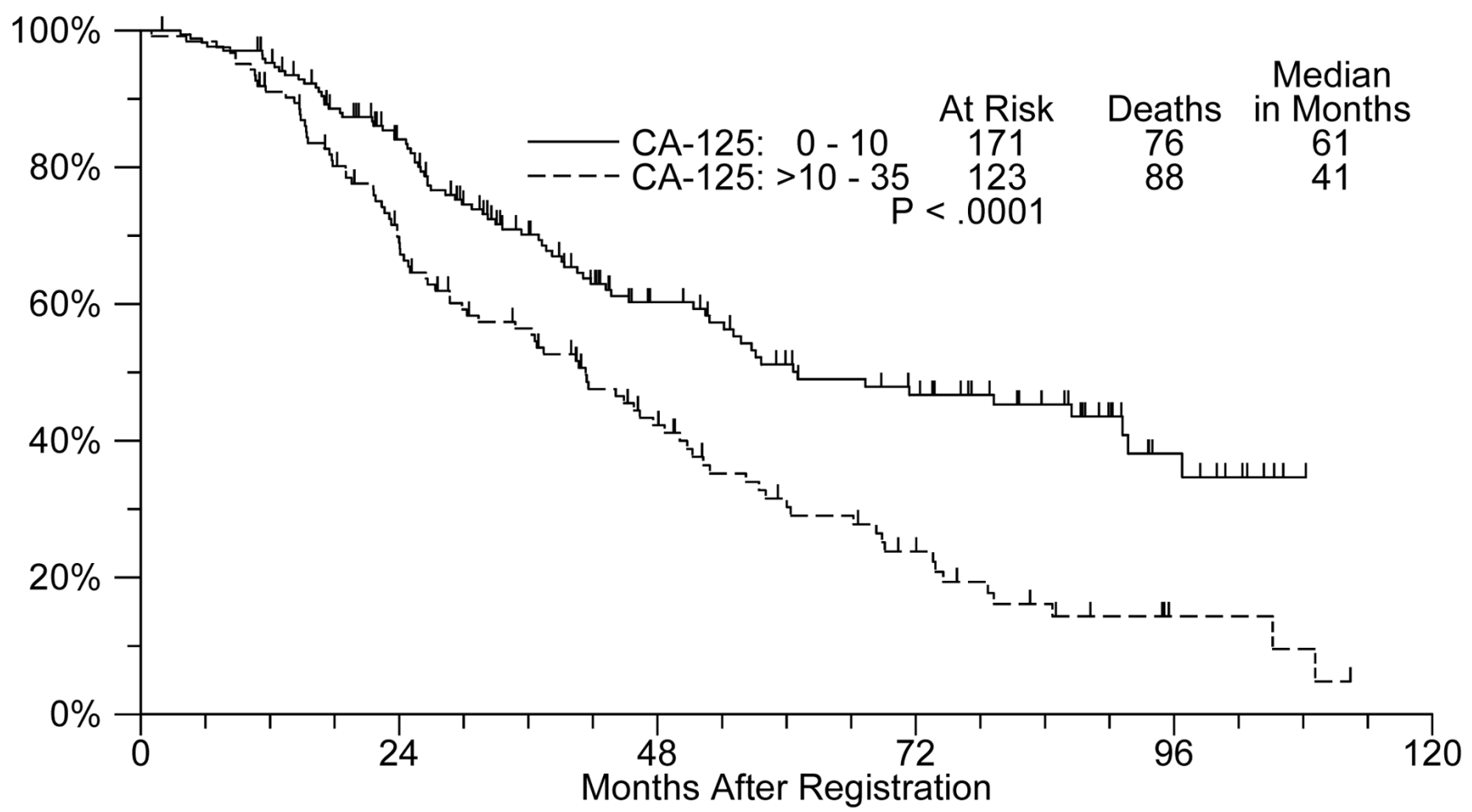

Figure 3.

Overall survival by CA-125 ( $\leq 10$ versus $>10-35)$ 
TABLE 1

TOXICITY

3-cycle arm $(n=136)$

12-cycle arm (n=149)

\begin{tabular}{|c|c|c|}
\hline & 3-cycle arm $(n=136)$ & 12-cycle arm $(n=149)$ \\
\hline Grade 4 hematologic & $13(10 \%)$ & $7(5 \%)$ \\
\hline Grade 2 neurologic & $20(15 \%)$ & $35(23 \%)$ \\
\hline Grade 3 neurologic & $1(1 \%)$ & $9(6 \%)$ \\
\hline Grade 3 pain & $1(1 \%)$ & $6(4 \%)$ \\
\hline
\end{tabular}

